\title{
An inversion involving the mouse Shh locus results in brachydactyly through dysregulation of Shh expression
}

\author{
Michael Niedermaier, ${ }^{1,2}$ Georg C. Schwabe,, ${ }^{1,3}$ Stephan Fees, ${ }^{4}$ Anne Helmrich, ${ }^{5,6}$ Norbert Brieske, ${ }^{1}$ \\ Petra Seemann, ${ }^{1}$ Jochen Hecht, ${ }^{1}$ Volkhard Seitz,, ${ }^{1}$ Sigmar Stricker, ${ }^{1}$ Gundula Leschik, ${ }^{6}$ \\ Evelin Schrock, ${ }^{5,6}$ Paul B. Selby, ${ }^{7}$ and Stefan Mundlos ${ }^{1,6}$

\begin{abstract}
${ }^{1}$ Max Planck Institute for Molecular Genetics, Berlin, Germany. ${ }^{2}$ Freie Universität Berlin, Fachbereich Biologie, Chemie, Pharmazie, Berlin, Germany. ${ }^{3}$ Department of Pediatric Endocrinology, Charité, Humboldt University, Berlin, Germany. ${ }^{4}$ Children's Hospital, University of Mainz, Mainz, Germany. 5Institute for Clinical Genetics, Technical University Dresden, Dresden, Germany. ${ }^{6}$ Institute for Medical Genetics, Charité, Humboldt University, Berlin, Germany. ${ }^{7}$ RiskMuTox, Oak Ridge, Tennessee, USA.
\end{abstract}

\begin{abstract}
Short digits $(D s h)$ is a radiation-induced mouse mutant. Homozygous mice are characterized by multiple defects strongly resembling those resulting from Sonic bedgehog $(S h b)$ inactivation. Heterozygous mice show a limb reduction phenotype with fusion and shortening of the proximal and middle phalanges in all digits, similar to human brachydactyly type A1, a condition caused by mutations in Indian hedgehog (IHH). We mapped Dsh to chromosome 5 in a region containing $S h b$ and were able to demonstrate an inversion comprising $11.7 \mathrm{Mb}$. The distal breakpoint is $13.298 \mathrm{~kb}$ upstream of $S h h$, separating the coding sequence from several putative regulatory elements identified by interspecies comparison. The inversion results in almost complete downregulation of Shb expression during E9.5-E12.5, explaining the homozygous phenotype. At E13.5 and E14.5, however, Shb is upregulated in the phalangeal anlagen of $D s h /+$ mice, at a time point and in a region where WT $S b h$ is never expressed. The dysregulation of $S h b$ expression causes the local upregulation of hedgehog target genes such as Gli1-3, patched, and Pthlh, as well as the downregulation of $I b h$ and Gdf5. This results in shortening of the digits through an arrest of chondrocyte differentiation and the disruption of joint development.
\end{abstract}

\section{Introduction}

Formation of the vertebrate appendicular skeleton involves a number of sequential phases resulting in the formation of the various tissues of the limb in a proximodistal sequence (reviewed in ref. 1). After the initial patterning phase, mesenchymal cells aggregate and differentiate into chondrocytes, forming the cartilaginous anlagen of the future skeleton. Mesenchymal condensations resemble the future skeleton in shape and size. In many instances, though, more than 1 bone or cartilage can arise from a single condensation. This is particularly evident in the limbs. The digital rays, consisting of the metacarpals/tarsals and 3 phalanges (proximal, middle, and distal) in digits II-V and 2 phalanges in digit I, are thought to be formed from prechondrogenic condensations that appear spatially continuous and subsequently segment into individual skeletal elements (1). The process of segmentation begins with a change in morphology of cells at the sites of joint formation. The cells become flattened, are more densely packed, lose their chondrogenic characteristics, and change their extracellular matrix composition $(2,3)$. In the chick, this so-called interzone develops into a 3-layered structure, with the central region undergoing apoptosis, resulting in the formation of the joint cavity (4).

A range of human skeletal dysplasias and mouse models indicate that joint formation is closely linked to proper chondrocyte differ-

Nonstandard abbreviations used: BAC, bacterial artificial chromosome; CNE, conserved noncoding element; Dsh, short digits; Dsh/+, Dsh heterozygote; EST, expressed-sequence tag; Hh, hedgehog; P1, proximal phalanx; P2, middle phalanx; P3, distal phalanx.

Conflict of interest: The authors have declared that no conflict of interest exists.

Citation for this article: J. Clin. Invest. 115:900-909 (2005)

doi:10.1172/JCI200523675 entiation and vice versa. Mutations in the genes for Indian hedgehog $(I H H)(5)$, growth and differentiation factor 5 (GDF5) (6), BMP-receptor 1B (BMPR1B) (7), and the receptor tyrosine kinase ROR2 $(8,9)$ have been shown to cause distinct subtypes of brachydactyly, a group of inherited disorders of the hands characterized by shortening of the digits together with abnormal phalangeal joint formation (10). Ihh, a secreted signaling peptide from the hedgehog $(\mathrm{Hh})$ family, is expressed in prehypertrophic chondrocytes. It activates Pthlh, which is expressed in periarticular cartilage, and inhibits the conversion of proliferating and prehypertrophic chondrocytes into hypertrophic chondrocytes, establishing a feedback loop between Ihh and Pthlh (11). The role of Ihh during cartilage differentiation and joint formation is underscored by the fact that mutations in $\mathrm{IHH}$ cause human brachydactyly type A1 (5) and $\mathrm{I} h \mathrm{~h}^{-/-}$mice exhibit shortened cartilage elements without joints (12). Thus, the cross-talk between Ihh and Pthlh is not only important for the regulation of chondrocyte differentiation in the growth plate but also essential for proper joint formation.

Another central molecule involved in the pathogenesis of brachydactyly is Gdf5, a signaling molecule from the TGF- $\beta$ superfamily expressed in the perichondrium and joint interzones of the developing appendicular skeleton (13). Lack of Gdf5 function in the mouse mutant brachypodism leads to shortening of limbs, brachydactyly, and loss of phalangeal joints (14), similar to the human acromesomelic dysplasias of the Grebe (15) and Hunter Thompson types (16) that are also caused by mutations in GDF5. Gdf5 signals through its receptor Bmpr1b. Mutations in BMPR1B lead to brachydactyly type A2 (7) and a subform of acromesomelic dysplasia (17). Mutations in ROR2, a receptor tyrosine kinase, result in brachydactyly type $\mathrm{B}$, a condition characterized by loss of distal 
A

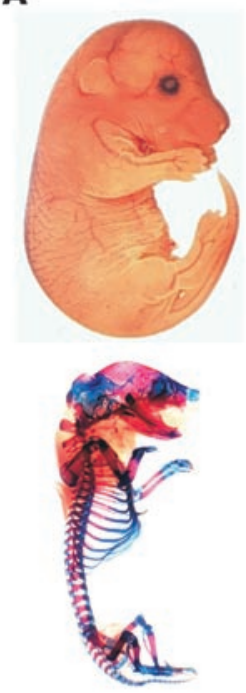

WT
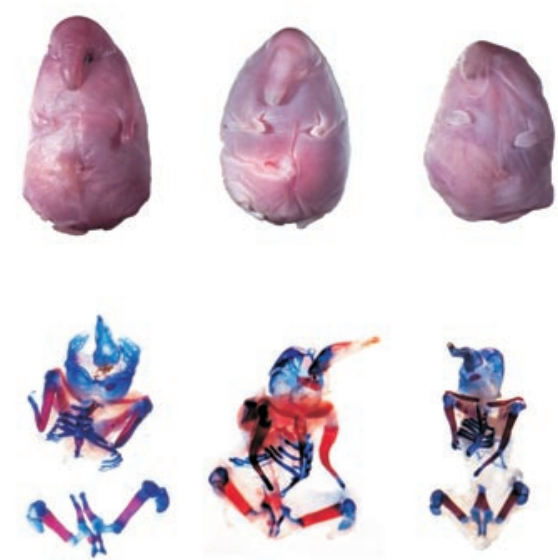

Dsh/Dsh

$D s h /+S_{S h}^{+/}$

$\mathrm{Shh}^{-{ }^{-}}$
B

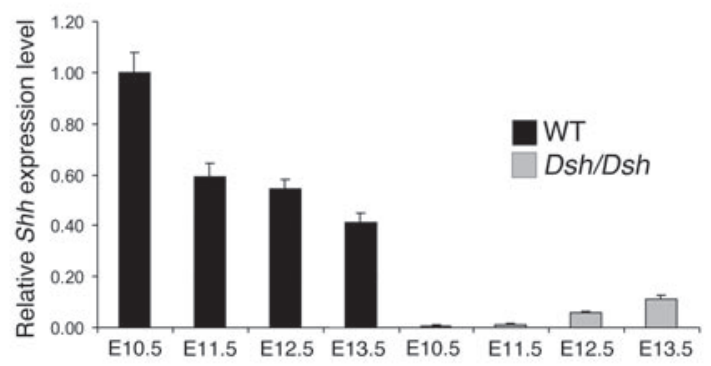

Figure 1

Dsh/Dsh a regulatory mutation of Shh. (A) Phenotype of E17.5 embryos. Alizarin red/alcian blue-stained skeletal preparations below. Dsh/Dsh, Dsh/+;Shh ${ }^{+-}$, and $S h h^{-/}$embryos show a nearly identical phenotype. (B) Quantitative RT-PCR from WT and Dsh/Dsh RNA samples obtained from E10.5, E11.5, E12.5, and E13.5 embryos. Bars represent levels $( \pm \mathrm{SD})$ of Shh expression relative to WT E10.5.

phalanges and symphalangism (9). The role of Ror2 in joint formation is less clear, but recent evidence points to a modulation of the Gdf5/BmpR1b pathway by Ror2 (18).

The above-mentioned disease-associated mutations alter the protein coding sequence of the gene in some way and thus cause the phenotype. However, there are potentially many other mechanisms that can disrupt normal gene function leading to pathological states. For example, chromosomal rearrangements such as inversions and translocations can interfere with normal gene expression by disrupting the promoter and transcribed exons from cis-acting regulators or by altering the chromatin structure adjacent to the gene (19). In these cases, the breakpoint can be located far away (up $1 \mathrm{Mb}$ ) from the gene. Such mutations are generally thought to result in a global downregulation of gene activity leading to haploinsufficiency and a loss-of-function phenotype. In this paper we present the mouse mutant short digits $(D s h)$ and its underlying molecular and developmental pathology. Ds $b$ is a semidominant mutant, lethal in the homozygote (20). We show that the $D s h$ phenotype is due to an inversion involving the Shb locus on chromosome 5. The inversion results in the downregulation of Shb expression during early stages of development, thus causing a
Shh loss-of-function phenotype in the homozygote. At later stages and in heterozygous animals, however, the inversion causes a misregulation of Shb expression in the limbs during morphogenesis of the phalanges, resulting in short digits and a brachydactyly phenotype. The $D s h$ mutant represents an as yet undescribed example of the temporal and spatial misregulation of gene expression due to a chromosomal rearrangement. The developmental pathology associated with this misexpression extends our understanding of the developmental pathology of brachydactyly.

\section{Results}

$D s h / D$ sh phenotype. The $D s h / D s h$ phenotype is characterized by multiple internal and skeletal defects (Figure 1A) (20). Dsh/Dsh embryos have a severe defect in midline patterning, and the musculature is virtually absent (21). Skeletal malformations are characterized by a complete lack of craniofacial bone, but the persistence of some cartilaginous tissues was evident in the craniofacial region. Furthermore, absence of vertebral bodies, dorsal ribs, and distal limb structures was observed. Scapula and humerus articulate, but the single bone that replaces radius and ulna is fused to the humerus. In the hindlimb, the femur is present and articulates to a tibia, which is truncated shortly after the joint. The distal limb structures (autopod) are absent in the hindlimbs and represented by a single, digit-like element in the forelimbs. The $D s h / D s h$ and $S h h^{-/-}$ phenotypes are very similar (22). However, E17.5 Dsh/Dsh mice are larger in size, and the proboscis in $D s h / D s h$ tends to be larger than in $S h h^{-/-}$mice. To test whether $D s h$ and $S h b$ are allelic, $S h h^{+/-}$mice were crossed with $D s h$ heterozygote $(D s h /+)$ mice and the double heterozygotes were compared with $D s h / D s h$ and $S h b^{-1-}$ mice. $D s h /+$; $\mathrm{Shb}^{+} /-$mice revealed a phenotype that was nearly identical to that of $S h h^{-1-}$ and $D s h / D s h$ mice, with the full spectrum of midline defects, internal anomalies, and limb malformations, indicating that $D s b$ and Shb are allelic and do not complement (Figure 1A).

In order to examine the expression of $S h b$ in $D s h / D s h$ embryos, quantitative RT-PCRs with total RNA from E10.5-E13.5 WT, Dsh/Dsh, $D s h /+$ and $S h h^{-/}$embryos were performed. The results show an almost complete loss of $S h b$ expression in $D s h / D s h$ embryos until E12.5. At E12.5 and E13.5, however, residual Shh expression is detectable. Quantification of Shb expression in comparison to the corresponding WT at E12.5 and E13.5 shows a reduction to $10 \%$ and 27\%, respectively (Figure 1B). At least some of the residual expression at E12.5 and E13.5 was shown by in situ hybridization to be due to ectopic expression of Shb in the proboscis of $D s h / D s h$ embryos (data not shown).

Mapping and mutation analysis. Our initial mapping showed linkage to proximal chromosome 5 within a region containing the Shb gene. In order to screen for mutations in the Shb cDNA and nearby regulatory elements, we PCR-amplified and sequenced the floor plate enhancer $10 \mathrm{~kb}$ upstream of $S h b$ and the $14-\mathrm{kb}$ genomic region comprising exons $1-3$, the promoter region, and the brain enhancer (Figure 2D) using cDNA and genomic DNA from $D s h / D s h$, C57BL10, C3H, and 101 mouse strains. No mutation was found. Several 101 strain polymorphisms were detected within the region, demonstrating that the original mutation had been induced in the 101-strain chromosome of the $(101 \times \mathrm{C} 3 \mathrm{H}) \mathrm{F}_{1}$ male that had been irradiated. The critical region was further refined using a mapping strategy with a Dsh/C57BL10 BALBc intercross. Twenty-five recombinants for D5Mit226 were identified, reducing the mapping interval at the centromeric margin. Six recombinant mice were identified for marker D5N13 at the telomeric margin still including the Shb gene (Figure 2A). 
A

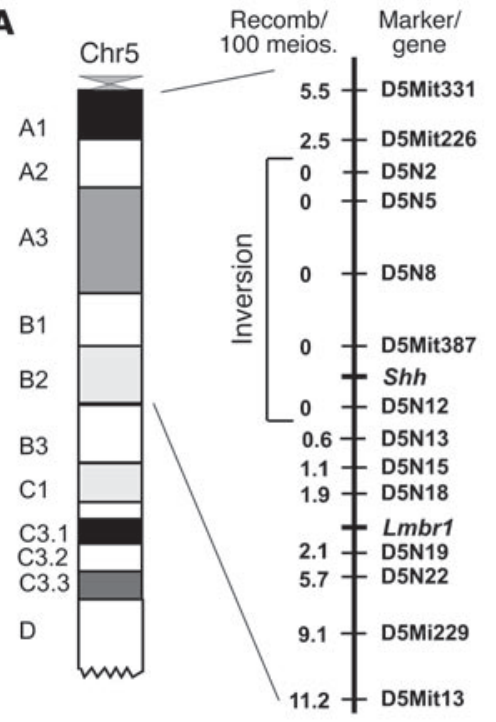

$\begin{array}{lr}\mathrm{Mb} & \mathrm{cM} \\ 4.2 & 3 \\ 14.1 & 5 \\ 15.1 & \\ 16 & \\ & \\ 23 & \\ & \\ 26.5 & 15 \\ 26.7 & \\ 26.9 & \\ 27 & \\ 27.1 & \\ 27.2 & \\ 27.4 & \\ 27.5 & \\ 28 & \\ 29.8 & 18 \\ & \end{array}$

34

20
B Dsh/Dsh 101

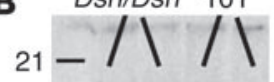

WT
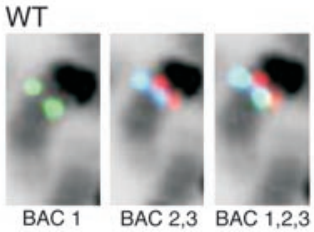

$D s h /+$

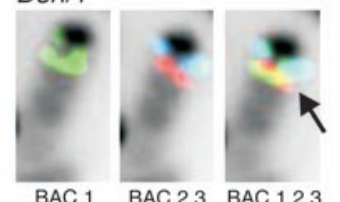

BAC 1 BAC 2,3 BAC 1,2,3 $930 \mathrm{~kb}$

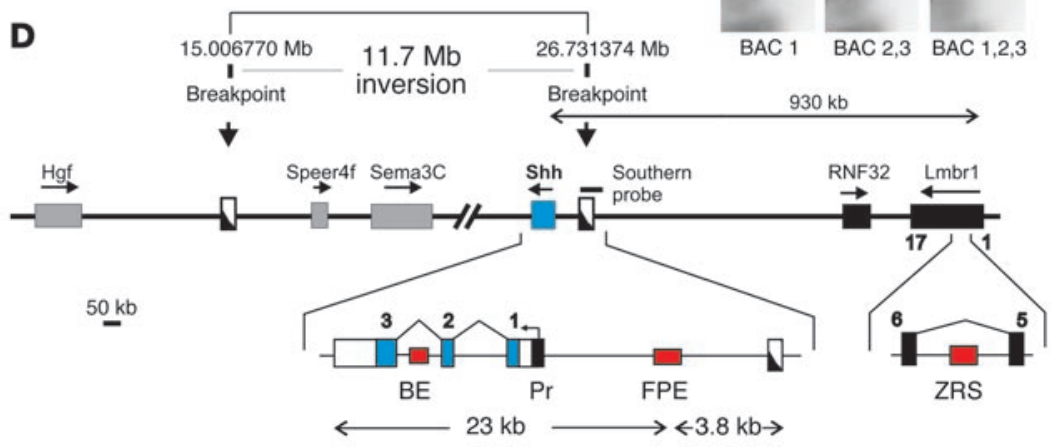

The large interval and the low number of recombinants were suggestive of a chromosomal rearrangement in the interval. To analyze such rearrangements with fluorescence in situ hybridization (FISH), 2 bacterial artificial chromosome (BAC) contigs covering both ends of the mapping interval were identified (chori, ensemble) and hybridized on interphase and metaphase spreads of spleen lymphocytes of $D s h /+$ mice (Figure 2C). Analysis of the hybridization experiments revealed a split signal for BAC RP2394F17 $(26.65-26.89 \mathrm{Mb})$ at the telomeric end of the region on one allele of $D s h /+$. In addition, the adjacent BAC RP24-507K16 (26.50-26.68 Mb) from the telomeric end and BAC RP23-130D17 $(15.21-15.41 \mathrm{Mb})$ from the centromeric end showed a reverse orientation on one allele of $D s h /+$. The detected inversion covers 11.7 $\mathrm{Mb}$ and includes the Shb gene.

To fine-map the breakpoints, Southern blot hybridizations using probes from a panel of PCR products residing within BAC RP23-94F17 at the telomeric end side was performed on DNA from WT (101) and Dsh/Dsh mice. We identified an aberrant restriction digest pattern in $D s h / D s h$ using a PCR probe located at $26.731011-26.731854 \mathrm{Mb}$ (Figure 2B). Subsequently, the telomeric breakpoint could be identified by genomic walking and was determined to reside at $26.731374 \mathrm{Mb}, 13.298 \mathrm{~kb}$ upstream of Shb and $3.874 \mathrm{~kb}$ upstream of the floorplate enhancer. BLAST analysis (http://www.ensembl.org/BLAST) of the inversion sequence
Identification of conserved elements. In order to identify conserved noncoding elements (CNEs) around the distal breakpoint, sequence comparisons were made with MultiPIPMaker and mVISTA programs using genomic DNA sequence from mouse, human, chick, and Fugu rubripes. This analysis revealed 4 (CNE1-CNE4) cross-species conserved elements distal of the breakpoint, making it highly likely that the $D s h$ phenotype is caused by a disruption of the Shb gene by long-range cis-acting elements (Figure 3).

The Dsh/+ limb phenotype is a model for human brachydactyly. Dsh/+ mice have short digits because of a fusion of the first and second phalanges of digits II- $\mathrm{V}$ and a shortened proximal phalanx of digit I (Figure 4). The carpal/tarsal bones and the remainder of the appendicular skeleton show no changes. Beginning at E14.5, chondrogenesis is delayed in the phalanges and metacarpals/tarsals of $D s h /+$ mice, as demonstrated by a lack of alcian blue staining (Figure 4C). By E15.5, the proximal (P1), middle (P2), and distal (P3) phalanges have formed in the WT and are separated by joints. At this time point, a P1/P2 fusion bone is present in $D s h /+$ mice that is not completely separated from the metacarpal by a joint. $\mathrm{P} 3$ is formed regularly. The P1 of digit I is split. In limbs of 4-week-old $D s h /+$ mice, digits II-V are severely shortened due to a fused P1/P2, and mild shortening of the metacarpals is present (Figure 4A). The P3 and the distal interphalangeal joint appear normal. In digit IV the metacarpophalangeal joint is fused. Fore- and hindlimbs are 

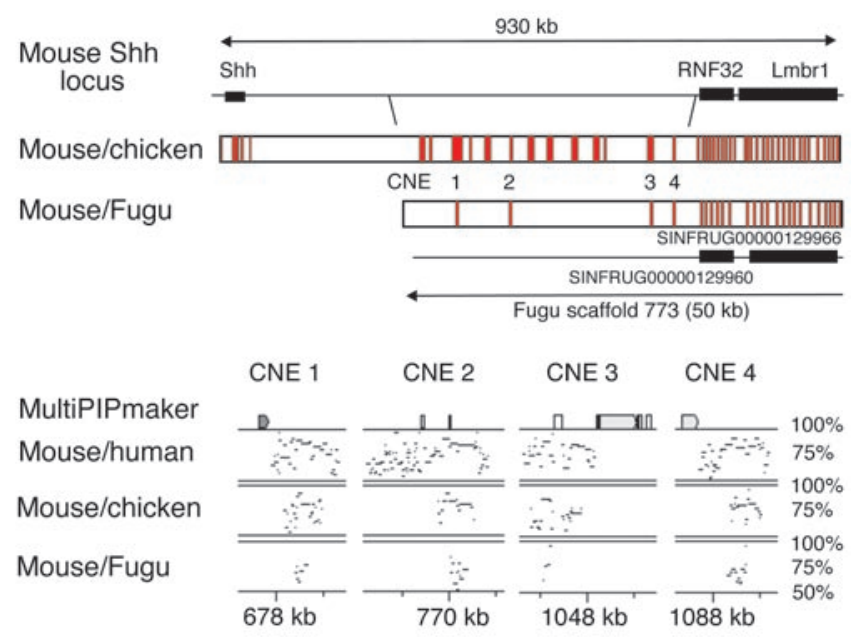

Figure 3

Sequence conservation at the Shh locus. Results of sequence alignments using MultiPIPMaker and mVISTA programs are summarized; red bars reflect high conservation between species. Four CNEs could be identified (CNE1-CNE4) that are highly conserved throughout the human, chick, and F. rubripes (Fugu) genomes. MultiPIPMaker plots of CNE1-CNE4 show regions of significant similarity between species as indicated by horizontal lines.

affected to the same extent. Therefore, the $D s h /+$ phenotype is very similar to human brachydactyly type A1.

H\&E-stained limb sections from E13.5-E16.5 mice were prepared for a more detailed analysis of chondrogenesis and joint formation (Figure 4D). In WT mice, differentiation of chondrocytes and formation of a joint interzone between the phalanges is starting at E13.5 and is clearly visible at E14.5. In the P1 and P2 skeletal elements of $D s h /+$ mice, chondrocytes remain undifferentiated, do not align in columns, and do not hypertrophy. Proliferation in $D s h /+$ limbs was analyzed with BrdU incorporation at E13.5-E16.5. At E14.5, BrdU-labeled cells are seen in prehypertrophic chondrocytes, the perichondrium, and the joint interzones. In $D s h /+$ digits, BrdU labeling is similar to WT in the perichondri$\mathrm{um}$, but is reduced in nondifferentiating chondrocytes and absent where joints should form (Figure 4E). TUNEL immunodetection in digits of WT mice showed apoptosis in the interdigital mesenchyme, the central layer of the 3-layered joint interzone. In $D s h /+$ mice, no apoptotic signals were detected, reflecting disturbed joint cavitation (Figure 4F). In addition, apoptosis in the interdigital web was reduced. Taken together, these findings indicate that the $D s h /+$ limb reduction phenotype is a result of delayed chondrogenesis, abnormal chondrocyte differentiation, reduced proliferation, and disruption of joint formation. The large number of proliferating cells in the area of the developing joints argues for the presence of a specific growth center in this region in the WT. The absence of this growth region is likely to contribute to the shortening of the individual phalangeal elements.

Shb expression in Dsh/+limbs. To investigate Shb expression in $D s h /+$ limbs, we performed a series of in situ hybridization experiments using a Shb-specific probe. Whole-mount in situ hybridization confirmed a normal expression pattern until E11.5 in the posterior mesenchyme (see Figure 6A). In situ hybridization of E13.5 and E14.5 on $D s h /+$ limbs showed ectopic expression of Shb in the cartilaginous anlagen of the developing digits (Figure 5A). In accordance with the $D s h /+$ phenotype, expression of Shb was observed only in the phalanges and not in the metacarpals or other structures of the developing limb. Hh signal is known to induce the expression of Pthlh over a distance in the joint region (11). Most likely as a result of ectopic Shb expression, Pthlh is ectopically expressed throughout the phalangeal cartilage anlage beginning at E13.5. Quantitative RT-PCR shows upregulation of Pthlh beginning at E13.5 (Figure $5 F)$. Ibh expression in $D s h /+$ mice showed a regular pattern in the metacarpal/tarsal anlagen and at the tip of the digits. However, we never observed $I h b$ expression in those elements that ultimately develop into the proximal and middle phalanges, the major site of the $D s h /+$ phenotype (Figure $5 C$ ). To determine the specificity of the $S h b$ probe and to rule out cross-hybridization with $I h b$, WT and $D s h /+$ E13.5 and E14.5 limbs were hybridized with Shb- and Ihbspecific probes. No staining was observed in WT limbs using the $S h b$-specific probe (Figure $5 \mathrm{~A}$ ), indicating that the signal observed in $D s h /+$ mice was specific. In contrast, hybridization with the $I h b$ specific probe gave a strong signal in the developing metacarpal and phalangeal anlagen in WT limbs, whereas $D s h /+$ limbs showed a signal only in the metacarpal but not the phalangeal anlagen (Figure 5C). Thus, the areas of Shb expression correlate with the region where $P t h l h$ is upregulated and $I h b$ is downregulated.

To determine whether Shb mRNA observed by in situ hybridization is translated, we performed immunohistochemistry with an antibody recognizing Hh proteins. We observed distinct staining of prehypertrophic chondrocytes in the WT metacarpals and phalanges, corresponding to Ihh expression. In the Dsh mutant, we observed regular staining in the metacarpals, corresponding to Ihh. However, in the phalanges, diffuse staining within the chondrocytes and in the flanking perichondrium was observed, corresponding to Shh protein (Figure 5C). These results are in good agreement with the in situ hybridization analysis.

Shb expression was further analyzed using quantitative RT-PCR (Figure 5D). At E10.5 and E11.5, Shb expression was reduced to approximately $50 \%$ of WT levels, as expected for a heterozygous regulatory mutation. Similar results were obtained in $\mathrm{Shb}^{+/-}$mice (data not shown). Shb expression is strongly downregulated in WT limbs at E12.5 and is practically switched off thereafter. A similar degree of downregulation was observed in the Dsh mutant at E12.5. However, in contrast to the WT, Shb expression was strongly upregulated at E13.5 and E14.5 in the mutant. Thus, the Dsh mutant behaves like a heterozygous loss-of-function mutation during the regular stages of Shb expression (i.e., E9.5-E11.5). Thereafter, Shb is turned off in the WT but strongly upregulated in the mutant.

Developmental analysis of Dsh/+limbs. To evaluate the effect of Shb misexpression, we analyzed the expression of Shh targets and other genes involved in digital patterning and initial chondrogenesis including Ptc, Gli1-3, Fgf4, Fgf8, Bmp2, Bmp4, Bmp7, Gremlin, Formin, Hoxd11-13, Gdf5, Nog, Col2a1, and Col10a1. In accordance with the normal expression of Shb at E10.5-E12.5 (Figure 6A), all tested genes displayed a regular expression pattern during the developmental stage at E10.5-E12.5 (Figure 6B). Concomitant with the upregulation of Shb at E13.5, we observed major differences in gene expression between WT and $D s h /+$ mice (Figure 6C).

Analysis of Hh downstream targets such as the Hh receptor Patched (Ptc) and Gli1 demonstrated ectopic expression throughout the putative joint region in Dsh/+ mice. Gli2 and Gli3, which have repressor activity and antagonize Gli1 and Shb function, are expressed in the joint and periarticular region (23). In $D s h /+$ limbs, Gli2 is expressed in the immature chondrocytes of the P1/P2 fusion 


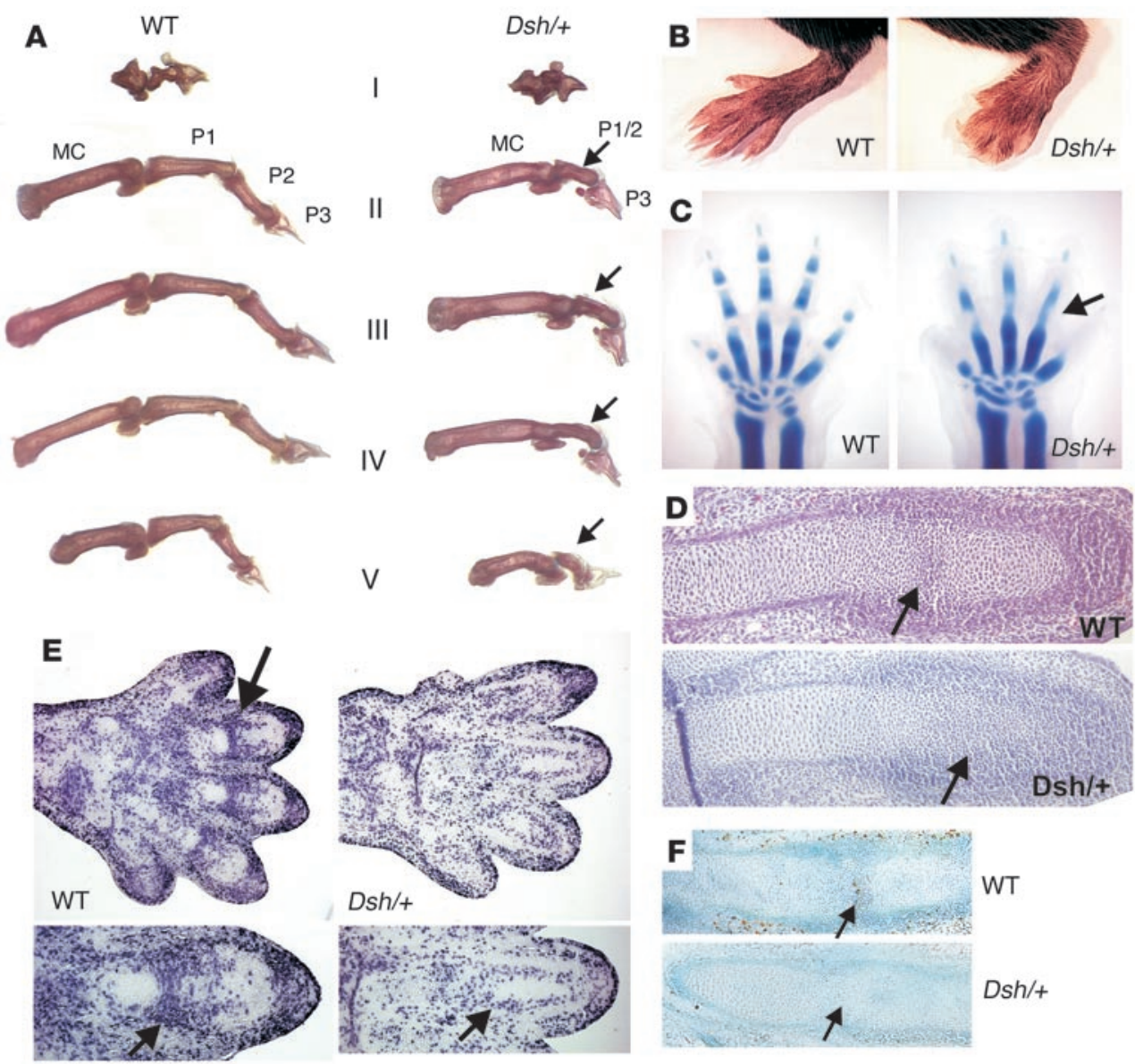

\section{Figure 4}

The Dsh/+ limb phenotype. (A) Alizarin red-stained skeletal preparations of 4-week-old WT and Dsh/+ forelimb digits. MC, metacarpals. Digits $\mathrm{I}-\mathrm{V}$ are shown. In Dsh/+ mice, the $\mathrm{P} 1$ and $\mathrm{P} 2$ elements are fused (P1/2, arrows) and severely reduced in size. In digit IV, the metacarpo-phalangeal joint is also fused. MC, metacarpals. (B) Hindlimb of WT and Dsh/+ adult mouse showing severe reduction in digit length. (C) Alcian blue staining of E15.5 WT and Dsh/+ limbs. Note lack of the middle phalanx anlage in all digits and persistent staining between the developing metacarpal and $\mathrm{P} 1$, indicating delayed or absent joint formation (arrow). (D) H\&E staining of E14.5 WT and Dsh/+ digits. WT shows the beginning of joint formation (arrow). In contrast, $D s h /+$ shows lack of joint interzone formation and undifferentiated chondrocytes in the region of prospective phalanges (arrow). (E) BrdU staining of E14.5 WT and Dsh/+ autopod (top) and digits (bottom) showing reduced proliferation in the region of joint formation (arrows). (F) TUNEL staining showing lack of apoptosis in joint interzones (arrow). Magnification, $\times 18(\mathbf{A}), \times 7$ (B), $\times 40(\mathbf{C}), \times 50$ (E), $\times 100$ (D and F). phalanx, and Gli3 expression is shifted from the joint to the perichondrium and the interdigital mesenchyme in the mutant.

Gdf5 is a signaling molecule from the TGF- $\beta$ superfamily that is expressed in early cartilage condensations, the perichondrium, and the joint interzones of the developing appendicular skeleton. In E13.5 digits of $D s h /+$ mice, Gdf5 is found in the perichondrium but does not show the characteristic striped pattern in the joint interzone. At E14.5, Gdf5 is expressed in a longitudinal stripe in the region where the putative joint would be established.

The differentiation of chondrocytes is delayed in $D s h /+$ digits, as shown by reduced collagen type II expression in early stages and absence of collagen type $\mathrm{X}$ expression, normally expressed in hypertrophic chondrocytes, in later stages.

\section{Discussion}

Proteins of the Hh family are key mediators of many fundamental processes in embryonic development (for review see ref. 24). Shh is the most prominent member of the Hh family of signaling molecules. It is involved in a variety of processes during development, malformation, and cancer. Loss of both Shb copies in mice leads to a severe midline defect and a variety of internal and skeletal malformations (22). In humans, heterozygous loss-of-function mutations in $\mathrm{SHH}$ result in holoprosencephaly, characterized by medial cleft lip and palate, single maxillary incisor, frontal CNS lobe fusion, and midfacial defects, including a spectrum ranging from hypotelorism to cyclopia $(25,26)$. Hh ligands elicit their effects by antagonizing the activity of their receptor patched (ptc), which binds a third protein, smoothened (Smo), a G protein-coupled receptor. The cubitus interruptus orthologs Gli1, Gli2, and Gli3 mediate the transcriptional control by $\mathrm{Hh}$ signaling in vertebrates, with Gli1 being an activator of Hh target gene activation, whereas Gli2 and Gli3 are considered to be repressors. Partial loss of this repressor activity underlies the various human and murine polydactylies that are caused by mutations in the Shb-Gli signal transduction pathway (for review see ref. 27).

$D s h$ is a regulatory Shb mutation. Homozygous $D s h / D$ sh embryos display a phenotype highly similar to the $\mathrm{Shb}^{-/}$phenotype. However, in contrast to $\mathrm{Shb}^{+/-}$mice, the $\mathrm{Dsh} /+$ mutant is characterized by short digits, a skull defect, and other anomalies (20). Using a positional cloning approach, we demonstrate that $D s h$ is caused by an intrachromosomal inversion that comprises $11.7 \mathrm{Mb}$. In accordance with the presence of an inversion, we observed a suppression of recombination in proximity to the breakpoints, a phenomenon thought to be due to the formation of inversion loops during meiosis causing unstable chromosomes. The inversion is likely to be caused by the initial irradiation experiment, because ionizing radiation frequently results in DNA double-strand breaks and genomic rearrangements (28).

One breakpoint of the $D s h$ inversion is $13 \mathrm{~kb}$ upstream of the Shb gene. Hence, the genomic rearrangement leaves the exons, the promoter, and 2 enhancers (29) intact. In humans, translocations $15-265 \mathrm{~kb} \mathrm{5'}$ of the $S H H$ promoter have been described that result in a holoprosencephaly phenotype similar to that observed in individuals with mutations in $\mathrm{SHH}$ (26). These findings can be interpreted as a long-range position effect due to position effect 
A

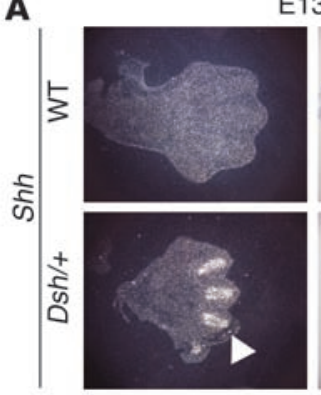

E13.5

B
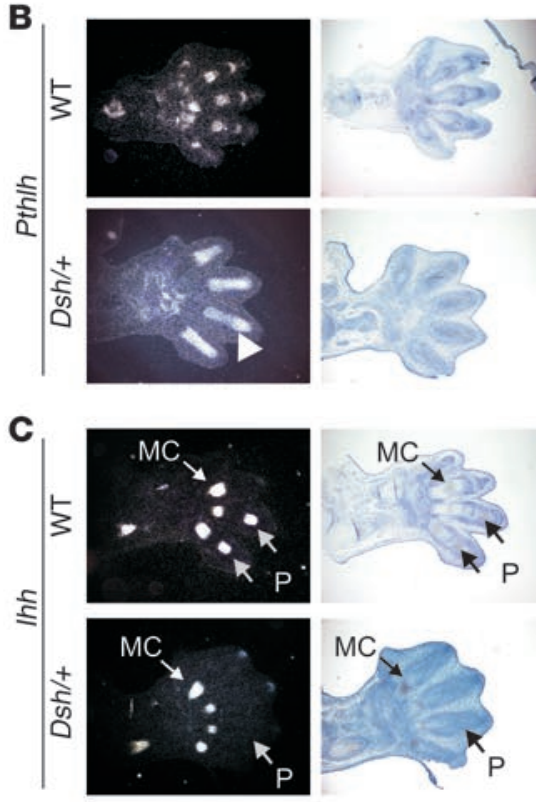

D
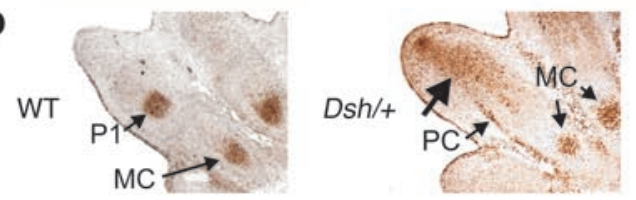

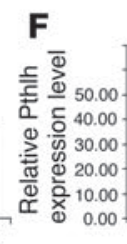

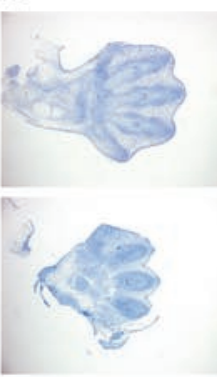
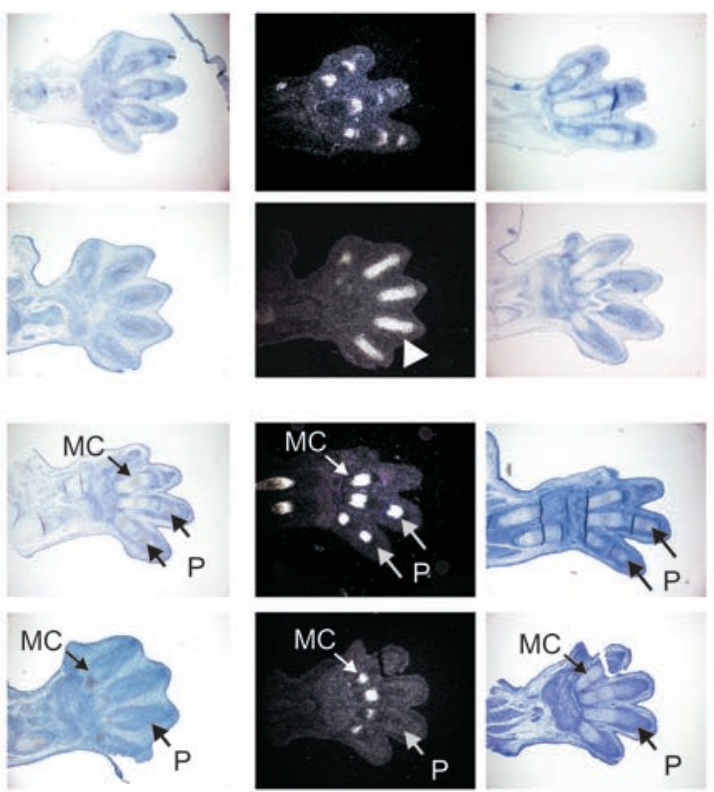

E14.5
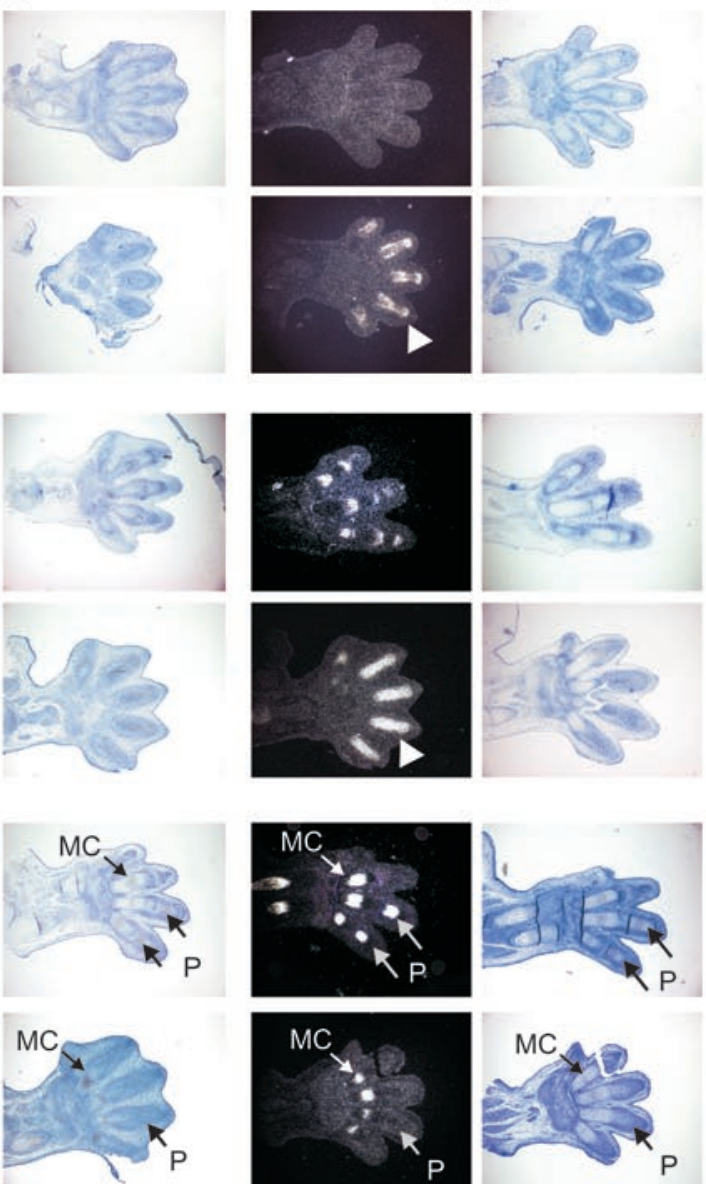

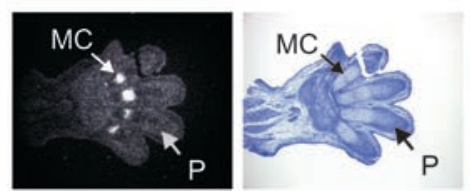

Pthlh expression in limbs
E Shh expression in limbs

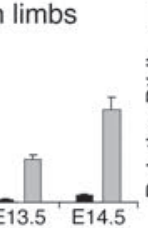$$
\text { . }
$$

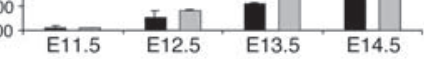

\section{Figure 5}

Shh expression in Dsh/+ limbs. (A) Section in situ hybridization at E13.5 and E14.5 showing strong expression of $S h h$ in the developing distal digits in Dsh/+ but not in WT limbs. Note that expression is restricted to the anlagen of the phalanges (arrowheads). At E14.5, expression is strongest in the perichondrium of the proximal phalanx. (B) Pthlh is strongly overexpressed in Dsh/+ throughout the entire phalangeal anlagen, whereas it is restricted to the ends of the anlagen in the WT. Note overlap with Shh expression as shown in (A). (C) Expression of Ihh in WT metacarpals and phalanges (P). In contrast, Ihh is not expressed in the phalangeal anlagen of $D s h /+$ limbs. (D) Immunohistochemistry with an antibody recognizing $\mathrm{Hh}$ protein shows restricted staining in prehypertrophic chondrocytes in the WT and Dsh/+ metacarpals and in WT phalanges corresponding to Ihh, but diffuse staining throughout the phalangeal anlagen in the mutant (large arrow) and in the perichondrium (PC) corresponding to Shh protein. (E and F) Quantitative RT-PCR of Shh (E) and Pthlh (F) mRNA in WT and Dsh/+ limbs at E10.5-E14.5. Bars represent levels $( \pm S D)$ of $S h$ expression relative to WT E10.5 and Pthlh expression relative to WT E11.5. Magnification, $\times 50$ (A-C), $\times 100$ (D).

malformations in $D s h / D s h$ mice that are indistinguishable from the $\mathrm{Shb}^{-/-}$phenotype. This suggested that $D s h$ and $S h b$ are allelic, a hypothesis supported by our complementation experiments. However, the situation is more complex, as demonstrated by the upregulation of Shb expression after E13.5. The Dsh inversion results not only in the downregulation of Shb expression but also in a dysregulation.

Experiments performed in transgenic zebrafish and mice indicate that Shb expression is controlled by multiple transcriptional regulators $(29,30)$. One Shb enhancer element was identified in intron 5 of the flanking $L m b r 1$ gene located approximately 1 $\mathrm{Mb}$ away from Shb. In the Ssq mouse mutant, this element (designated ZRS) is disrupted (31), resulting in the ectopic expression of Shb at the anterior margin of the limb in addition to its normal posterior expression domain, and, consequently, in a polydactyly phenotype. Point mutations in this element segregate with polydactyly in 4 unrelated postaxial polydactyly families (31) and in the mouse mutant hemimelic extratoes $(H x)$ (32). Using a genetic test variegation, a phenomenon describing the variable but heritable inhibition of gene expression due to an altered chromatin environment through a chromosomal rearrangement (19). However, the presence of multiple conserved sites upstream of the Shb gene, as shown by our interspecies sequence comparisons (Figure 3), is highly suggestive of the presence of locus control regions. Such sites have been found to be associated with a number of tightly controlled genes and were shown to confer position-independent, copy number-dependent expression of a linked gene. The separation of the promoter/transcription unit from such elements can be expected to lead to reduction or absence of $S h b$ transcription. As shown by quantitative RT-PCR, this is the case, at least during the early stages of development. The almost complete loss of Shb expression explains the severe midline defect and the associated with the $S s q, H x$, and $S h b^{-/-}$alleles, it was elegantly shown that the ZRS element is a cis-acting regulator $(31,33)$. Against the background of these findings, a 6-kb deletion including exon 4 and the surrounding intronic sequence of the of the human $\mathrm{Lmbr} 1$ homolog C2ORF7 in acheiropodia, a malformation syndrome with a limb truncation phenotype similar to that seen in the $\mathrm{Shb}^{-/-}$mice (34), may be interpreted as loss of another Shb enhancer element, possibly belonging to an enhancer repressor complex. In addition to the regulatory elements discussed, other sites must exist that control the ZPA-specific expression of Shb, as shown in the mouse mutant replicated anterior zeugopod (raz) (35) and the chick mutant oligozeugodactyly (ozd) (36). Similar to $D s h$, raz is caused by an inversion involving the Shb locus. The breakpoints, however, are not well defined and appear to reside $12 \mathrm{Mb}$ distal of Lmbr1 and $3 \mathrm{Mb}$ proxi- 
A

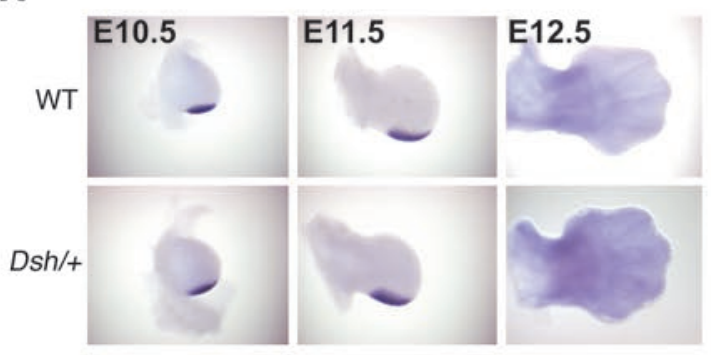

B
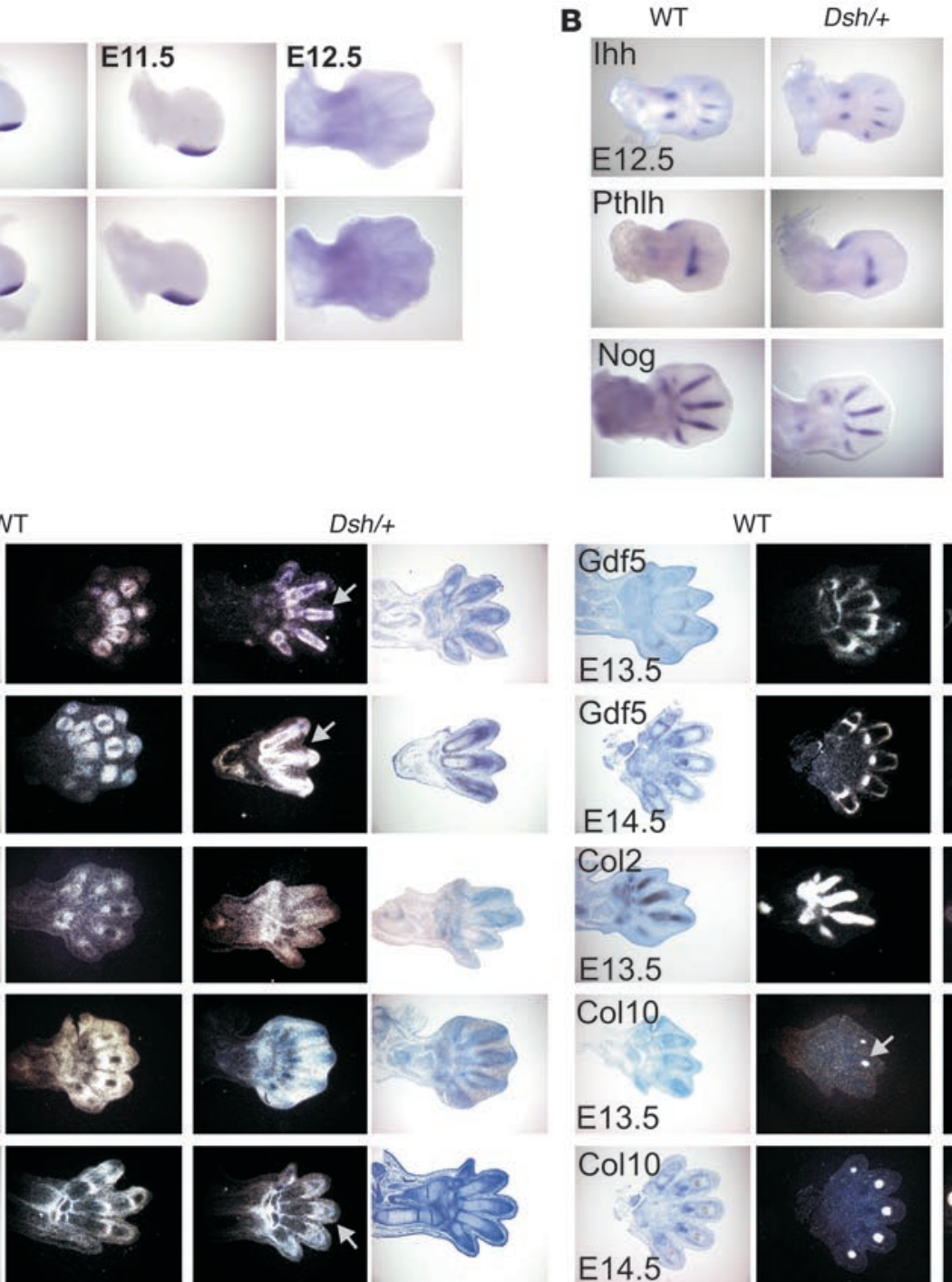
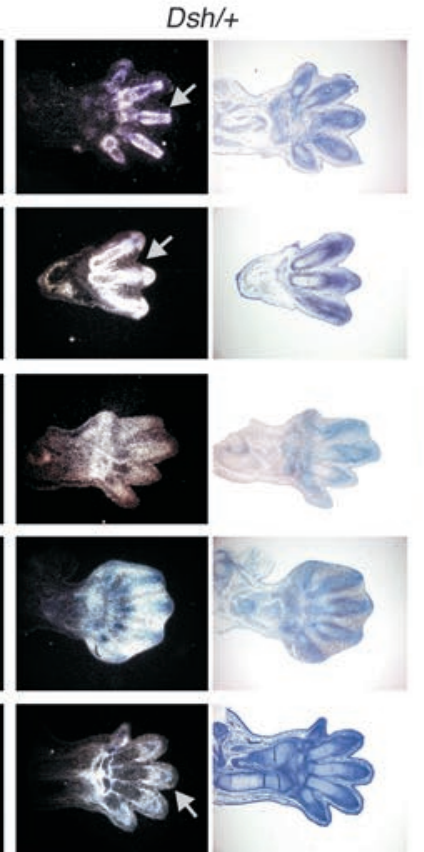

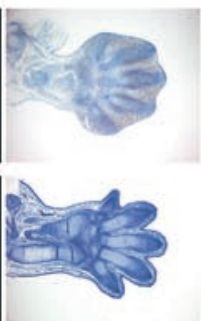

WT

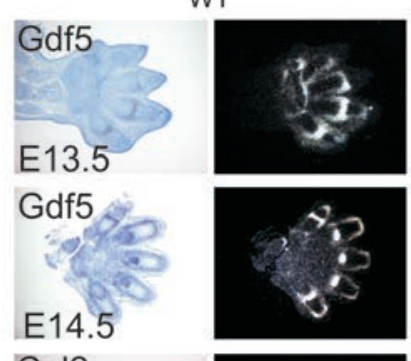

Col2

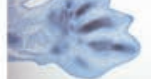

E13.5

Col10
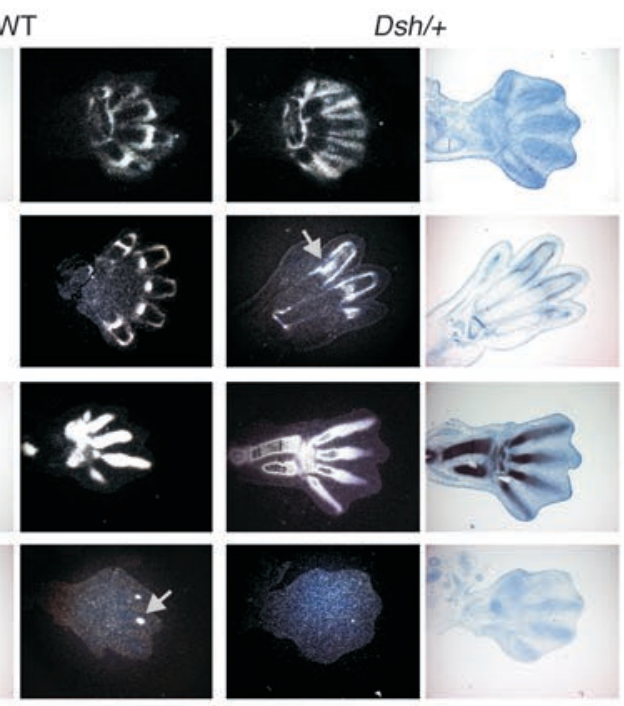

E13.5

Col10

E14.5
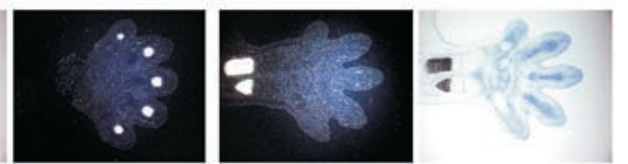

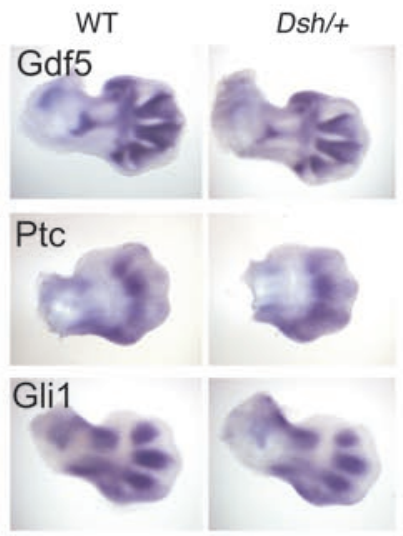

Figure 6

Developmental analysis of $D s h /+$ limbs. Expression of marker genes relevant for digit development was performed using whole-mount (A and B) and section (C) in situ hybridization. (A) Expression of Shh in the posterior limb region and downregulation at E12.5 showing no difference between WT and Dsh/+. At E12.5 (B), we find normal patterns of expression for all genes tested. At E13.5 and E14.5 (C), the majority of genes show an abnormal pattern. Note misexpression of Hh receptor patched (ptc) and downstream effectors Gli1-Gli3 (arrows). Gdf5 first shows lack of expression in the developing joints (E13.5) and is then (E14.5) expressed in a more longitudinal fashion in the area of the future metacarpophalangeal joints (arrow). Col2a1 (Collagen type I/ a1; Col2) is expressed throughout the phalangeal anlagen but is less pronounced in the distal regions of $D s h /+$ digits. Lack of Col10a1 (Collagen type $X$ a1; Col10) expression in digit anlagen demonstrates delay in chondrocyte differentiation in Dsh/+. Magnification, $\times 50(\mathbf{A}-\mathbf{C})$.

mal of Shb. Raz and ozd result in the downregulation of Shb expression in the limb and a successive loss of $S h b$ target gene expression, suggesting that they interfere with a ZPA-specific enhancer. Such elements may reside within the conserved sequences identified in this study that are located between the $D s h$ breakpoint and the $L m b r 1$ gene. The different phenotypes in acheiropodia, $D s h$, and $S s q$ suggest that activating and/or repressing properties may be located near the ZRS. In addition to removing an enhancer element from the genomic context necessary for proper expression of $S h b$, the $D s h$ inversion could also eliminate an element involved in silencing Shb. The loss of this putative silencer would then result in ectopic Shb expression. In addition, $L m b r 1$ may be involved in Shb activation, as
Lmbr1/- mice with no phenotype show loss of posterior digits when crossed with mice carrying a deletion of chromosome 5 including Shb (37). Retroviral overexpression of Lmbr1 in chick limbs, however, has no effect (38).

Other possibilities to consider are the disruption of a second gene involved in the regulation of Shb expression in trans or the translocation of a new promoter element before the Shb gene resulting in the misexpression of $S h b$ via this element. A detailed analysis of the genes and expressed-sequence tags (ESTs) located in the vicinity of the centromeric breakpoint revealed no gene spanning a breakpoint, and no differences in the expression of nearby located genes were observed, making the hypothesis of a second gene unlikely. 


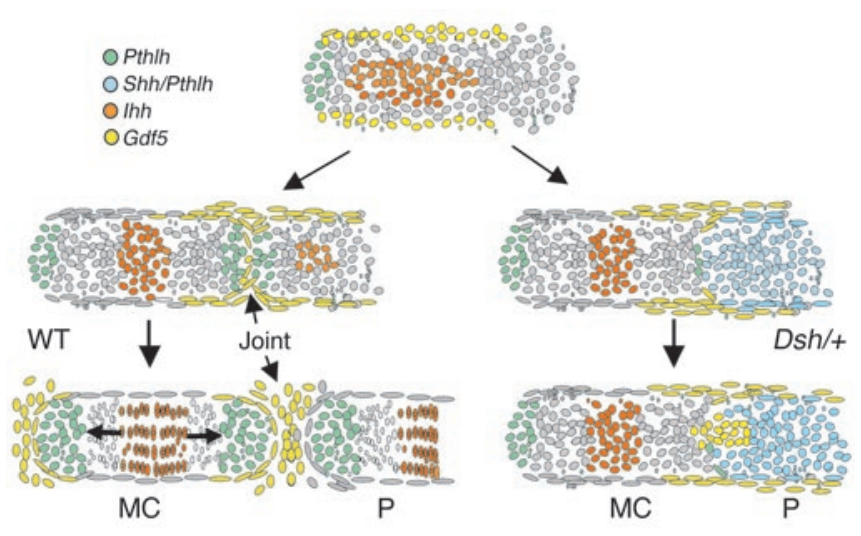

Figure 7

Digit development and joint formation. Schematic of WT (left) and Dsh/+ (right) digit and joint development and gene expression patterns corresponding to E12.5 (top), E13.5 (middle), and E14.5 (bottom). The expression domains of Ihh, Pthlh, Gdf5, and Shh are indicated. Ihh induces $P$ thlh at a distance and thereby regulates the distance to the developing joint space (arrows). Gdf5 is first expressed around the condensing cells of the cartilaginous anlage (E12.5) and then in the future joint space, where it regulates cell recruitment and proliferation. In $D s h /+\operatorname{limbs}$, this process is disrupted after E13 by expression of Shh in the cartilaginous anlagen and the perichondrium of the future P1, thereby inducing Pthlh and repressing Ihh and Gdf5. In contrast, Shh is not expressed in the metacarpals. This results in a fusion of $\mathrm{P} 1$ and $\mathrm{P} 2$, an impediment in the formation of the metacarpophalangeal joint, and a delayed chondrocyte differentiation and bone formation in the metacarpals.

Furthermore, our complementation experiments strongly argue against this possibility. None of the tested ESTs or genes showed an expression pattern compatible with the ectopic expression of $S h b$ in $D s h /+$ mice, rendering unlikely the possibility that the inversion puts $S h b$ under the control of a new regulatory element that might alter its normal expression pattern. However, we cannot completely rule out this possibility, because the chromosomal region around the proximal breakpoint is not fully characterized.

$D$ sh is a model for abnormal joint formation. We propose that the brachydactyly phenotype in $D s h$ is caused by the reactivation of $S h b$ expression in the perichondrium and cartilage of the digital anlagen. The misexpression of Shb results in an upregulation of Shh targets and, consequently, in the disruption of the Ihh-Pthlh feedback loop and other signaling pathways involved in joint development, such as the BMP/Gdf5 pathway (Figure 7). Owing to the late onset of misexpression, the phenotype is temporally and spatially restricted to the development of the proximal and middle phalanges and thus does not show a typical Shb-related phenotype such as polydactyly or oligodactyly. Consistent with this hypothesis, we can show normal expression of molecular markers for limb patterning and cartilage development until E12.5 in the Dsh/+ digits.

The major players known to be involved in the organogenesis of phalanges and the interphalangeal joints are Ihh, the Ihh-Pthlh regulatory loop, and Gdf5. As shown by overexpression in the chick and gene inactivation experiments in mice, Ihh regulates chondrocyte differentiation in the growth plate, joint formation, and osteoblast differentiation in endochondral bones $(11,12)$. Mutations in $I H H$ cause the human conditions acrocapitofemoral dysplasia (39) and brachydactyly type A1 (5). At E13.5, Ihb expression condenses in the middle of the metacarpal anlagen and starts to be visible first in the P1 and then in the P2. In $D s h /+$ mice the metacarpal expression domain is maintained, but expression in the proximal and middle phalanges is absent. In spite of the missing Ibh expression, Hh downstream genes such as Gli1 and ptc are highly expressed, suggestive of a Hh signal not detected by our I $\mathrm{hb}$ specific probe. Searching for such a signal, we identified Shb expression in the phalangeal anlagen of $D s h /+$ digits. Shh, Ihh, and the third member of the Hh family, Desert hedgehog (Dhh), are highly homologous, use the same receptors and signal transduction pathways, and are able to replace one another in appropriate in vitro and in vivo models (40). It is therefore conceivable that Shh elicits the same response, possibly with variable potency, as Ihh, when expressed in cartilaginous tissue. We propose that the ectopic expression of Pthlh as well as the loss of Ibh expression in the phalangeal anlagen of $D s h /+$ mice is induced by $S h b$ overexpression.

In the WT mouse, Pthlh is induced at a distance from the Hh signal, enabling the formation of a growth plate architecture with graded levels of chondrocyte differentiation. This pattern is completely disrupted in $D s h /+$ phalanges, resulting in the absence of a regular growth plate and the persistence of chondrocytes in an undifferentiated state. Consistent with our hypothesis is the finding that overexpression of Pthlh in chondrocytes using a collagen type II promoter leads to a delay in mineralization and chondrocyte maturation (41). Likewise, addition of the active $\mathrm{NH}_{2}$ terminal fragment of $S h b$ in limb cultures and overexpression of $I h b$ by retroviral gene transfer in chick limbs both delay hypertrophy of chondrocytes by upregulation of Pthlh at the periarticular joint region $(11,42)$. Conse-

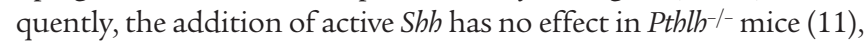

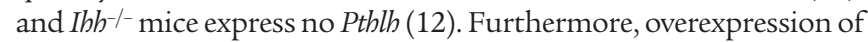
Hh (Ihh) in chick embryos results in the downregulation of endogenous $I h h$, probably by the upregulation of Pthlh (11). Similar results were obtained in the mouse when overexpressing Ihh or Shh in chondrocytes $(43,44)$, supporting our observation that $I h b$ is downregulated in areas of $\mathrm{Shb}$ misexpression.

These findings indicate that the perturbation of the Ihh-Pthlh feedback loop in $D s h /+$ is responsible for the delay in chondrocyte differentiation and hypertrophy, resulting in shortening of the $\mathrm{P} 1$ and P2. In addition to the delay in chondrocyte differentiation, Pthlh overexpression and disruption of the physical distance between $I h b$ and Pthlh expression domains can be expected to interfere with the segmentation process. Gdf5 has a key role in joint formation, as demonstrated by the absence of interphalangeal joints in the brachypodism mouse, which lacks functional Gdf5 (14). The lack of Gdf5 expression at the appropriate sites is expected to contribute strongly to the $D s h /+$ phenotype.

The Hh antagonists Gli2 and Gli3 also show a major expression domain in the developing joint, whereas Gli1 is spared from the joint and expressed around the cartilage anlage. Gli1 shows an identical ectopic expression as $S h b$ in $D s h /+$, underscoring its role as transcriptional activator and primary mediator of $\mathrm{Hb}$ function. Conversely, Gli2 and Gli3 have repressor activity and antagonize $H h$ and Gli1 function (23). In $D s h /+$ limbs, they have lost their normal pattern and are expressed throughout the autopod. Because the Gli genes are direct downstream targets of $\mathrm{Hh}$ signaling, their abnormal expression is likely to be induced by the ectopic Shb expression observed in $D s h /+$ limbs. Misexpression of Gli1, Gli2, Gli3, and Gdf5 either directly or indirectly disrupts the intricate process necessary for joint formation and patterning and thus further contributes to the process. Inactivation of $I b h$, as in the Ihb knockout mouse, as well as Gdf5 in the brachypodism mouse leads to the loss of joints. $I h b$ is not expressed in digits of brachypodism mice, and Gdf5 is upregulated in the peri- 
chondrium of $\mathrm{Ihb}^{-/-}$mice (data not shown), suggesting that Ihh and Gdf5 may regulate each other's expression. The hypothesis presented here is supported by the finding that overexpression of Shb in chick limbs also results in the loss of joints (45), as in $D s h /+$ digits.

The developmental analysis of digits and joints in the $D s h /+$ mutant indicates that the formation of phalangeal elements and joints mutually depend on each other. The interplay of Gdf5, Gli2, Gli1, and Pthlh in the joint space with Ihh expressed in the middle of the anlage is likely to influence the spacing of joints and the size of the phalangeal elements (Figure 7). In this model, the developing joint region serves as a secondary signaling center that coordinates the size and number of cartilage elements by regulating proliferation, cell recruitment, chondrocyte differentiation, and, finally, joint formation. Human and mouse phenotypes with hypoplasia/aplasia of phalanges and/or abnormal interphalangeal joint formation are likely to be caused by a disruption of this process. Furthermore, this study shows that chromosomal rearrangements can result in unexpected misregulation of gene expression during development, a mechanism that may occur more frequently than previously thought.

\section{Methods}

Mice. Dsh is an autosomal semidominant mutation that was initially found in one of the progeny of $\mathrm{a}(101 \times \mathrm{C} 3 \mathrm{H}) \mathrm{F}_{1}$ hybrid male that had been exposed to 600 Röntgen $(\mathrm{R})+600 \mathrm{R}$ of $96 \mathrm{R} / \mathrm{min} \mathrm{x}$-ray irradiation, with an 8-week interval between exposures (20). The mutant was subsequently kept on the $\mathrm{C} 3 \mathrm{H} / 101$ background and was then crossed onto a C57BL10 background. Genotype analysis was performed by PCR amplification of extraembryonic membranes or tails using the D5Mit72 microsatellite marker, which is polymorphic for 101 and C57BL10 backgrounds and is located within the $D s h$ linkage interval on mouse chromosome 5. PCR was performed in $50-\mu 1$ reactions containing $1.5 \mathrm{mM} \mathrm{MgCl}_{2}$ and $2 \mathrm{pmol} / \mu \mathrm{l}$ of each primer. PCR-amplified DNA products of sizes $132 \mathrm{bp}$ for C57BL10 and $140 \mathrm{bp}$ for 101 were visualized on $4 \%$ ethidium bromide-stained agarose gels. Shb ${ }^{+/-}$ mice served for complementation assay with $D s h /+$ and were genotyped as described elsewhere (22). This study was performed according to the guidelines of the ethics committee of the Landesamt für Arbeitsschutz, Gesundheitsschutz und technische Sicherheit, Berlin, Germany.

Linkage analysis and fine mapping. Linkage of $D s h$ was established by crossing $D s h /+$ females with Mus castaneus males. $\mathrm{F}_{1}$ mice that showed the $D s h /+$ phenotype were intercrossed. One hundred and nine mice from the $\mathrm{F}_{2}$ generation with a $D s h /+$ phenotype served for linkage analysis using a genomewide set of microsatellite markers. In a second step, a fine-mapping analysis was performed by crossing $D s h /+$ females with $\mathrm{BALB} / \mathrm{c}$ males. This cross generated 950 mice with a $D s h /+$ phenotype that were tested using publicly available polymorphic markers and markers generated using the Tandem Repeats Finder program version 2.02 (http://tandem.bu.edu/). Labeling of the markers was performed with a single-reaction nested PCR method using a fluorescent dye-labeled M13(-21) primer (46) and analyzed on the ABI 3100 Prism Genetic Analyzer (Applied Biosystems).

Identification of breakpoints. Three-color FISH was performed on metaphase chromosomes and nuclei of $D s h /+$ splenocytes in accordance with standard procedures. BACs were labeled with Biotin-16-dUTP, Digoxigenin-11-dUTP (Roche Diagnostics Corp.), or Tamra-dUTP (Applied Biosystems) (red). Biotin-16-dUTP was visualized with FITC (green) and Digoxigenin-11-dUTP with Cy5 (far red). Fluorescent signals were documented with an epifluorescence microscope (Leica DMRXA) equipped with a cooled charge-coupled device camera (Photometrics) and bandpass filters (Chroma Technology Corp.). The QFISH-software (Leica Microsystems) was used for the analysis of the FISH results. Southern blot analysis was performed according to standard protocols with BamHI-,
EcoRV-, PstI-, EcoRI-, and HindIII-digested DNA and analyzed using a PhosphorImager system (Molecular Dynamics). The genomic region of the Shb gene was amplified with long-range PCR (Roche Diagnostics Corp.) from the mouse strains $D s h / D s h$, C57BL10, C3H, and 101; cloned into TopoXL Kit (Invitrogen Corp.); sequenced; and analyzed on an ABI 3100 sequencer (Applied Biosystems). Genomic walking was performed using GenomeWalker Kit (Clontech). All sequences were annotated according to ENSEMBL Mouse genome v 23.32c.1.

Skeletal preparations, in situ hybridization, and histological analysis. Timed matings were performed for the analysis of the mutant phenotype, and skeletal preparations were prepared as described elsewhere (47). Histology, whole-mount and ${ }^{33} \mathrm{P}$ in situ hybridization was performed as previously reported (48). Dsh/+ and WT embryos at E10.5, E11.5, and E12.5 served for whole-mount hybridizations. Limbs of E13.5-E14.5 mice were used for section in situ hybridization. The following riboprobes were used: $A l x 4, B m p 2$, Bmp4, Bmp7, Col2a1, dHand, Fgf4, Fgf8, Gdf5, Gli1, Gli2, Gli3, Hoxa11, Hoxd11, Hoxd12, Hoxd13, Ihh, Noggin, Ptc, Pthlh, Pthr1, and Shb (47-49).

BrdU incorporation into DNA strands was used to analyze proliferation of chondrocytes as described previously (48). Apoptosis was analyzed with the TUNEL assay using ApopTag kit (Intergen) in accordance with the manufacturer's protocol. Immunohistochemistry was performed with an anti-Shh antibody (ShhAb80) as described previously (50).

Quantitative RT-PCR. Quantitative PCR was performed from RNA extracted from limb buds and whole embryos. Limb buds of E10.5-E14.5 WT and $D s h /+$ embryos were removed, collected in RNA-later (Qiagen) and genotyped. For RNA extraction, a minimum of 10 limbs were pooled, minced with an Ultra-Turrax T8, and extracted using Trizol (Invitrogen Corp.). For whole embryos, E10.5-E13.5 Dsh/Dsh, Dsh/+ and Shb $h^{-/}$embryos were collected and extracted with Trizol. One microgram of RNA of each sample was transcribed to cDNA (Taqman Reverse Transcription Reagents; Applied Biosystems). PCR was performed in a 30- $\mu 1$ reaction containing $10 \mu \mathrm{l}$ 1:10 diluted cDNA or standard, $5 \mu \mathrm{l}$ primer mix (7.5 pmol of each primer), and $15 \mu \mathrm{l}$ SYBR green PCR master mix (Applied Biosystems). PCR was performed in triplicate on an Abi Prism 7900 HT Sequence Detection System. Relative quantification of gene expression was performed using the standard curve method as described by the manufacturer (User Bulletin \#2, 10/2001; Applied Biosystems).

Comparative sequence analysis. Sequence alignments between the species mouse, human, chicken, and F. rubripes were made with the MultiPIPMaker and mVISTA programs $(51,52)$ through their websites (http://pipmaker. bx.psu.edu/cgi-bin/multipipmaker and http://www-gsd.lbl.gov/vista/). Sequences of the various genomes used for alignments were obtained from ENSEMBL (http://www.ensembl.org).

\section{Acknowledgments}

This work was supported by a grant from the Deutsche Forschungsgemeinschaft to S. Mundlos. We thank C. Chiang for kindly providing us with $\mathrm{Shb}^{+/-}$mice and A. McMahon for supplying us with anti-Shh antibody.

Received for publication October 19, 2004, and accepted in revised form January 25, 2005.

Address correspondence to: Stefan Mundlos, Max Planck Institute for Molecular Genetics, Ihnestrasse 73, D-14195 Berlin, Germany. Phone: 49-30-8413-1267; Fax: 49-30-8413-1385; E-mail: mundlos@molgen.mpg.de.

Michael Niedermaier and Georg C. Schwabe contributed equally to this work. 
1. Mariani, F.V., and Martin, G.R. 2003. Deciphering skeletal patterning: clues from the limb. Nature. 423:319-325.

2. Archer, C.W., Morrison, H., and Pitsillides, A.A. 1994. Cellular aspects of the development of diarthrodial joints and articular cartilage. J. Anat. 184:447-456.

3. Pitsillides, A.A., Archer, C.W., Prehm, P., Bayliss, M.T., and Edwards, J.C. 1995. Alterations in hyaluronan synthesis during developing joint cavitation. J. Histochem. Cytochem. 43:263-273.

4. Mitrovic, D.R. 1977. Development of the metatarsophalangeal joint of the chick embryo: morphological, ultrastructural and histochemical studies. Am. J. Anat. 150:333-347.

5. Gao, B., et al. 2001. Mutations in IHH, encoding Indian hedgehog, cause brachydactyly type A-1. Nat. Genet. 28:386-388.

6. Polinkovsky, A., et al. 1997. Mutations in CDMP1 cause autosomal dominant brachydactyly type C. Nat. Genet. 17:18-19.

7. Lehmann, K., et al. 2003. Mutations in bone morphogenetic protein receptor $1 \mathrm{~B}$ cause brachydactyly type A2. Proc. Natl. Acad. Sci. U. S. A. 100:12277-12282.

8. Oldridge, M., et al. 2000. Dominant mutations in ROR2, encoding an orphan receptor tyrosine kinase, cause brachydactyly type B. Nat. Genet. 24:275-278.

9. Schwabe, G.C., et al. 2000. Distinct mutations in the receptor tyrosine kinase gene ROR2 cause brachydactyly type B. Am. J. Hum. Genet. 67:822-831.

10. Kornak, U., and Mundlos, S. 2003. Genetic disorders of the skeleton: a developmental approach. Am.J.Hum. Genet. 73:447-474.

11. Vortkamp, A., et al. 1996. Regulation of rate of cartilage differentiation by Indian hedgehog and PTH-related protein. Science. 273:613-622.

12. St-Jacques, B., Hammerschmidt, M., and McMahon, A.P. 1999. Indian hedgehog signaling regulates proliferation and differentiation of chondrocytes and is essential for bone formation. Genes Dev. 13:2072-2086.

13. Francis-West, P.H., et al. 1999. Mechanisms of GDF-5 action during skeletal development. Development. 126:1305-1315.

14. Storm, E.E., et al. 1994. Limb alterations in brachypodism mice due to mutations in a new member of the TGF beta-superfamily. Nature. 368:639-643.

15. Thomas, J.T., et al. 1996. A human chondrodysplasia due to a mutation in a TGF-beta superfamily member. Nat. Genet. 12:315-317.

16. Thomas, J.T., et al. 1997. Disruption of human limb morphogenesis by a dominant negative mutation in CDMP1. Nat. Genet. 17:58-64.

17. Demirhan, O., et al. 2005. A homozygous BMPR1B mutation causes a new subtype of acromesomelic chondrodysplasia with genital anomalies. J. Med. Genet. In press.

18. Sammar, M., et al. 2004. Modulation of GDF5/BRI-b signaling through interaction with the tyrosine kinase receptor Ror2. Genes Cells. 9:1227-1238.
19. Kleinjahn, D.A., and van Heyningen, V. 2005. Longrange control of gene expression: emerging mechanism and disruption in disease. Am.J. Hum. Genet. 76:8-32.

20. Selby, P.B., Bolch, S.N., Mierzejewski, V.S., McKinley, T.W., Jr., and Raymer, G.D. 1993. Synergistic interactions between two skeletal mutations in mice: individual and combined effects of the semidominants cleidocranial dysplasia (Ccd) and short digits (Dsh). J. Hered. 84:466-474.

21. Kruger, M., et al. 2001. Sonic hedgehog is a survival factor for hypaxial muscles during mouse development. Development. 128:743-752.

22. Chiang, C., et al. 1996. Cyclopia and defective axial patterning in mice lacking Sonic hedgehog gene function. Nature. 383:407-413.

23. Ruiz i Altaba, A. 1999. Gli proteins encode contextdependent positive and negative functions: implications for development and disease. Development. 126:3205-3216.

24. McMahon, A.P., Ingham, P.W., and Tabin, C.J. 2003. Developmental roles and clinical significance of hedgehog signaling. Curr. Top. Dev. Biol. 53:1-114.

25. Roessler, E., et al. 1996. Mutations in the human Sonic Hedgehog gene cause holoprosencephaly. Nat. Genet. 14:357-360.

26. Belloni, E., et al. 1996. Identification of Sonic hedgehog as a candidate gene responsible for holoprosencephaly. Nat. Genet. 14:353-356.

27. Schwabe, G.C., and Mundlos, S. 2004. Genetics of congenital hand anomalies. Handchir. Mikrochir. Plast. Chir. 36:85-97.

28. Woychik, R.P., et al. 1990. Molecular and genetic characterization of a radiation-induced structural rearrangement in mouse chromosome 2 causing mutations at the limb deformity and agouti loci. Proc. Natl. Acad. Sci. U. S. A. 87:2588-2592.

29. Muller, F., et al. 1999. Intronic enhancers control expression of zebrafish sonic hedgehog in floor plate and notochord. Development. 126:2103-2116.

30. Jeong, Y., and Epstein, D.J. 2003. Distinct regulators of Shh transcription in the floor plate and notochord indicate separate origins for these tissues in the mouse node. Development. 130:3891-3902.

31. Lettice, L.A., et al. 2002. Disruption of a long-range cis-acting regulator for Shh causes preaxial polydactyly. Proc. Natl. Acad. Sci. U. S. A. 99:7548-7553.

32. Lettice, L.A., et al. 2003. A long-range Shh enhancer regulates expression in the developing limb and fin and is associated with preaxial polydactyly. Hum. Mol. Genet. 12:1725-1735.

33. Sagai, T., et al. 2004. Phylogenetic conservation of a limb-specific, cis-acting regulator of Sonic hedgehog (Shh). Mamm. Genome. 15:23-34.

34. Ianakiev, P., et al. 2001. Acheiropodia is caused by a genomic deletion in C7orf2, the human orthologue of the Lmbr1 gene. Am. J. Hum. Genet. 68:38-45.

35. Krebs, O., et al. 2003. Replicated anterior zeugopod (raz): a polydactylous mouse mutant with lowered Shh signaling in the limb bud. Development. 130:6037-6047.
36. Ros, M.A., et al. 2003. The chick oligozeugodactyly (ozd) mutant lacks sonic hedgehog function in the limb. Development. 130:527-537.

37. Clark, R.M., et al. 2001. Reciprocal mouse and human limb phenotypes caused by gain- and lossof-function mutations affecting Lmbr1. Genetics. 159:715-726.

38. Maas, S.A., and Fallon, J.F. 2004. Isolation of the chicken Lmbr1 coding sequence and characterization of its role during chick limb development. Dev. Dyn. 229:520-528.

39. Hellemans, J., et al. 2003. Homozygous mutations in IHH cause acrocapitofemoral dysplasia, an autosomal recessive disorder with cone-shaped epiphyses in hands and hips. Am. J. Hum. Genet. 72:1040-1046.

40. Pathi, S., et al. 2001. Comparative biological responses to human Sonic, Indian, and Desert hedgehog. Mech. Dev. 106:107-117.

41. Weir, E.C., et al. 1996. Targeted overexpression of parathyroid hormone-related peptide in chondrocytes causes chondrodysplasia and delayed endochondral bone formation. Proc. Natl.Acad. Sci.U.S.A. 93:10240-10245.

42. Lanske, B., et al. 1996. PTH/PTHrP receptor in early development and Indian hedgehog-regulated bone growth. Science. 273:663-666.

43. Minina, E., et al. 2001. BMP and Ihh/PTHrP signaling interact to coordinate chondrocyte proliferation and differentiation. Development. 128:4523-4534.

44. Tavella, S., et al. 2004. Targeted expression of SHH affects chondrocyte differentiation, growth plate organization, and Sox9 expression. J. Bone Miner. Res. 19:1678-1688.

45. Merino, R., et al. 1999. Expression and function of Gdf-5 during digit skeletogenesis in the embryonic chick leg bud. Dev. Biol. 206:33-45.

46. Schuelke, M. 2000. An economic method for the fluorescent labeling of PCR fragments. Nat. Biotechnol. 18:233-234.

47. Mundlos, S. 2000. Skeletal morphogenesis. Methods Mol. Biol. 136:61-70.

48. Albrecht, A.N., et al. 2002. The synpolydactyly homolog (spdh) mutation in the mouse - a defect in patterning and growth of limb cartilage elements. Mech. Dev. 112:53-67.

49. Bitgood, M.J., and McMahon, A.P. 1995. Hedgehog and Bmp genes are coexpressed at many diverse sites of cell-cell interaction in the mouse embryo. Dev. Biol. 172:126-138.

50. Gritli-Linde, A., Lewis, P., McMahon, A.P., and Linde, A. 2001. The whereabouts of a morphogen: direct evidence for short- and graded long-range activity of hedgehog signaling peptides. Dev. Biol. 236:364-386

51. Schwartz, S., et al. 2000. PipMaker--a web server for aligning two genomic DNA sequences. Genome Res. 10:577-586.

52. Mayor, C., et al. 2000. VISTA: visualizing global DNA sequence alignments of arbitrary length. Bioinformatics. 16:1046-1047. 